\title{
Justicia distributiva, responsabilidad y compensación
}

\author{
GUSTAVO PEREIRA \\ Universidad de la República (Uruguay)
}

RESUMEN. La distinción realizada por Dworkin entre persona y circunstancias le ha permitido fundar un fuerte criterio para delimitar aquello que debería ser compensado de aquello que no. Las críticas a esta distinción han develado una insuficiencia que me lleva a presentar un criterio más preciso para discriminar lo compensable de lo que no lo es. Tal criterio se basa en la distinción entre autonomía potencial y autonomía plena, siendo lo compensable todo aquello que le permitiría a un individuo alcanzar el estado de autonomía plena. La compensación se realizaría en término de mínimos y dicho criterio oficiaría como guía para una posible extensión del mecanismo del mercado hipotético de seguros de Dworkin.

Ronald Dworkin, en su propuesta de igualdad de recursos, ha presentado una concepción de justicia distributiva que puede ser resumida en dos principios de justicia. El primer principio demanda que las personas en cualquier momento de sus vidas tengan diferentes conjuntos de riqueza como resultado de las elecciones que hayan realizado. El mercado es presentado como el mecanismo idóneo para llevar adelante esta distribución, porque a través de este mecanismo los costos del trabajo, bienes o inversiones son determinados en base al deseo que tengan los otros ciudadanos de pagar por ese bien. El segundo principio requiere que las personas no tengan diferentes conjuntos de riqueza solamente porque poseen diferentes dotacio-
Abstract. The distinction drawn by Dworkin between a person and his circumstances has enabled him to found a strong criterion to discriminate what should be compensated from what should not. Criticism to this distinction has revealed an insufficiency which has led me to present a more precise criterion. My criterion is based upon the distinction between potential and full autonomy, so the lack of something that would let an individual reach the level of full autonomy should be compensated. Compensation should be made in terms of minimums, and this criterion could work as a guide to a possible extension of Dworkin's mechanism of the hypothetical insurance market.

nes naturales que les permiten producir lo que los otros desean, o porque son favorecidos por la suerte. Por lo tanto, los resultados del mercado deben ser corregidos para contrarrestar los efectos de la suerte o de la dotación natural ${ }^{1}$. Este segundo principio utiliza el mecanismo del mercado hipotético de seguros, por el cual se asegura a los afectados compensaciones en términos de mínimos.

De estos dos principios se sigue, de acuerdo con la igualdad de recursos, que el Estado debería compensar a aquellos afectados por circunstancias que se encuentran más allá de su control, por ejemplo, una discapacidad, pero no por aquello que depende de las elecciones de los individuos, por ejemplo, la preferencia 
por ciertos vinos. Una distinción muy significativa refuerza esto último; la misma es entre la persona y sus circunstancias, en base a esta distinción todo lo que se encuentra bajo la órbita de la persona, es decir, preferencias, creencias, etc., es responsabilidad del sujeto $\mathrm{y}$, por lo tanto, no es compensable. A su vez, todo lo que se encuentra bajo la órbita de las circunstancias se sitúa más allá de la decisión y elección del sujeto, y en virtud de ello el sujeto no es responsable y debería ser compensado.

Algunos autores ${ }^{2}$ han centrado el foco de sus críticas en esta distinción entre la persona y sus circunstancias. Al igual que el propio Dworkin, consideran, siguiendo a Aristóteles ${ }^{3}$, central la diferencia entre las acciones de una persona que son causadas por aquellas circunstancias que están más allá de su control, y aquellas por las cuales son responsables. Por lo tanto, el acuerdo también estaría en que la sociedad debería indemnizar a las personas que tiene pobres resultados que son consecuencia de causas que se encuentran más allá de su control, pero que, en cambio, no debería hacerlo con aquellos resultados de causas que se encuentran bajo su control. Los puntos de discordancia están en dónde y cómo trazar la línea que divide aquello de lo que un sujeto es responsable de lo que no.

\section{I}

Arneson trata el problema de dónde establecer la línea divisoria que permita determinar cuándo un sujeto es responsable de una cierta acción y, en consecuencia, de cuándo tendría derecho a compensación, realizando una importante crítica a Dworkin ${ }^{4}$ y a su defensa de que los sujetos son enteramente responsables por sus preferencias.

En la visión de Arneson, el sostener que alguien es responsable por sus preferencias es completamente falso, si es que esto significa que tales preferencias se han desarrollado hasta el estado actual debido a factores que se encuentran absolutamente bajo el control de los afectados. Pero esto cambia si se reconoce que, si bien nuestras actuales preferencias han sido adquiridas a través de procesos que estaban más allá de nuestro alcance, ahora serían controlables, en el sentido de que podrían realizarse acciones que cambiaran nuestras preferencias en la forma en que aspiramos. En esta interpretación, el ser responsable de nuestras preferencias es compatible con la compensación de aquellas preferencias difíciles de satisfacer, hasta el momento en que éstas sean readaptadas y los involucrados adquieran el mismo nivel de satisfacción de preferencias que tienen los otros 5 .

En base a esto es que el corte entre preferencias y circunstancias no sería conceptualmente correcto, ya que una persona podría no ser enteramente responsable de sus preferencias, y, por lo tanto, si se mantiene esta distinción tendríamos que hablar de distintos grados o niveles de responsabilidad a nivel de preferencias. En cambio, si se adoptara la distinción oportunidad/logros, entonces podría darse cuenta de la responsabilidad de una forma más apropiada, colocando la responsabilidad y la no responsabilidad a cada uno de los lados de esta distinción, porque de lo que una persona no es responsable es de sus oportunidades, mientras que sí lo es de los logros que pueda alcanzar.

Cohen coincide a grandes rasgos con la propuesta de Arneson, y como crítica a los igualitaristas a nivel de recursos, y en particular a Dworkin, realiza una distinción que le servirá para explicar las limitaciones que tendrían estas otras teorías igualitaristas al compensar cierto tipo de casos. La distinción es relevante en situaciones en las que los individuos padecieran algún tipo de afección física y hubiera que examinar su caso para una posible com- 
pensación, la misma es dificultad/costo. Para ilustrarla digamos que se puede encontrar alguien para quien no es dificultoso mover sus brazos, ya que puede hacerlo perfectamente, pero sí es muy costoso hacerlo, debido a que estos movimientos le provocan fuertes dolores. Un ejemplo puede contribuir a la explicación: transportar a alguien hasta el aeropuerto de su ciudad en la parte trasera de su bicicleta es muy dificultoso, pero no es costoso porque esa persona disfruta enormemente los desafíos. Por otra parte, es costoso, pero no dificultoso extenderle un cheque por 50.000 pesetas a alguien ${ }^{6}$.

Volviendo al caso del hombre para quien es costoso mover su brazo debido al dolor que siente al hacerlo, supongamos que existiera una medicina cara que pudiera suprimir tal dolor. Los igualitaristas transferirían los recursos necesarios para que este individuo adquiriera tal medicamento, pero el punto de Cohen es que, si bien esto requeriría compensación, no puede ser representado como una compensación por discapacidad, porque la capacidad de nuestro individuo para mover su brazo está intacta. En este tipo de casos existe una dimensión de bienestar que es irreductible a recursos. La crítica se encuentra en que un ejemplo como el que se ha manejado excede a la órbita de recursos y se asienta en la de bienestar, por lo que dentro de la propuesta de Dworkin esta necesidad extra de compensación caería bajo la categoría de gustos caros.

Esta crítica pasa por alto una distinción que hace Dworkin, aquella que se da entre lo que puede ser entendido como un gusto caro para un individuo, y aquello que es una discapacidad y, por lo tanto, requiere compensación. Esta distinción, como ya he indicado, se basa en otra más básica, y es entre la persona y sus circunstancias; todo aquello que hace a la persona se encuentra bajo su control; por lo tanto, toda preferencia que desarrolle un sujeto estará fuera de lo que es compensable, ya que el sujeto es responsable del cultivo y desarrollo de tal preferencia. Esta distinción es reforzada en una reciente respuesta de Dworkin a Cohen ${ }^{7}$, en la que apela a la distinción persona/circunstancias al proponer la distinción elección/suerte, donde la responsabilidad se asienta a nivel de la persona que lleva adelante diferentes elecciones, pero no a nivel de las circunstancias o la fortuna. Dentro del primer campo son ubicables los gustos caros, mientras que las circunstancias hacen a aquello que se encuentra más allá de su control y que, por lo tanto, requeriría de compensación, por ejemplo, tener una discapacidad.

El problema se genera en aquellos aspectos que se encuentran en la zona límite entre preferencias y circunstancias; por ejemplo, la conducta de fumar puede ser atribuida tanto a la órbita de la personalidad como a la de las circunstancias, y para discernir las dificultades que se dan en estos casos es que Dworkin toma como criterio último al propio agente. Para ello sostiene que todos aquellos hábitos y conductas que preferiría no tener y contra los que se aseguraría en el mercado hipotético de seguros, son circunstancias o más específicamente discapacidades, mientras que no lo son aquellos que sí prefiriese continuar teniendo ${ }^{8}$. Esto permite discernir entre un gusto caro y una circunstancia que dificulta el llevar adelante un plan de vida, y que, por lo tanto, necesita compensación, aunque estemos hablando de la misma conducta. Lo que la coloca de un lado u otro de la línea es la forma en cómo la vive el propio afectado, mediada por la objetivación que posibilita el mercado hipotético de seguros.

Con respecto a casos como el del individuo que sufre de un fuerte dolor al mover sus brazos, debe decirse que Dworkin también acordaría en tal compensación, ya que esta característica es una clara desventaja para el sujeto que le demanda mayores recursos para poder llevar adelante su plan de vida. 
La crítica más importante, y que creo realmente cuestiona los fundamentos de la propuesta de Dworkin, es la que Cohen y Arneson hacen con respecto a la posibilidad de elección y construcción de las preferencias que tiene un individuo.

Volviendo a la distinción entre una persona y sus circunstancias, puede decirse que Dworkin coloca a las preferencias dentro de la esfera de la persona y a las capacidades en la esfera de las circunstancias. Lo que se encuentra al centro de esta distinción entre una persona y sus circunstancias es la sugerencia de que las personas forman sus preferencias, pero no así sus capacidades. La distinción circunstancias/persona es asimilable a la formado/no formado, donde en el ámbito de la persona se encontraría lo no formado, y, por lo tanto, la presencia de la elección, mientras que en el ámbito de las circunstancias se encontraría lo formado, y, por lo tanto, la ausencia de elección.

Según Cohen, tal distinción revela que Dworkin ha pasado por alto el conjunto de preferencias que se encuentran en el trasfondo de creencias que tiene todo sujeto; esto hace que ciertas elecciones se encuentren bajo la órbita del sujeto, pero que no todas lo estén ${ }^{9}$. Esta distinción que equipara el par formado/no formado con el par circunstancias/persona, es una distinción dogmática, ya que en ambos lados de la línea es posible encontrar tanto lo formado como lo no formado.

El poco atractivo que tiene la distinción formado/no formado hace que se vuelva la atención a la distinción circunstancias/persona como ámbito en el que se presenta también lo elegido y lo no elegido. Creo que al cuestionar esta distinción, se puede reconocer que a nivel de la persona existen algunas características que son producto de su elección y otras que no lo son, y que a nivel de las circunstancias se da la misma situación.
Esto nos conduce a considerar la distinción que realiza Cohen entre elecciones genuinas y aquellas que no lo son. Aquí se generaría una dificultad que sería encontrar un criterio para diferenciar lo genuino de las elecciones. Cohen ${ }^{10} \mathrm{y}$ Arneson ${ }^{11}$ sostienen que la información manejada por los sujetos es central para diferenciar un tipo de elección de otra, pero aclaran que para la justicia es suficiente con la información más relevante. Habría un umbral a partir del cual los sujetos serían responsables de sus elecciones, es decir, un umbral a partir del cual harían elecciones genuinas, y hasta tanto se lo supere los sujetos no son responsables de sus elecciones y, por lo tanto, pueden llegar a ser compensados.

Ante esto, Dworkin ${ }^{12}$ responde que es inconcebible sostener que un individuo pueda vivir sus creencias, ambiciones o juicios como afortunados o desafortunados accidentes. Este planteamiento hace que no sea posible distinguir en estos casos entre discapacidades y creencias, gustos, convicciones, etc. De acuerdo con Dworkin, las preferencias no son concebibles como estímulos que provocan una simple respuesta sensorial, sino que toda preferencia se encuentra inmersa en juicios de valor independientes. Por lo tanto, la preferencia por alguna actividad no se encuentra en que provoque una estimulación sensorial, sino que se asienta en que tal práctica es juzgada como valiosa. De ahí que Dworkin considere imposible tratar como discapacidades de las que no se es responsable a las preferencias que se encuentran formadas de esta manera. Bajo nuestras convicciones, gustos y preferencias razonamos debido a que éstas se encuentran fuertemente entrelazadas con nuestros juicios, y en tanto que son producto de un proceso racional que controlamos es que somos responsables por sus consecuencias. Este proceso hace que nuestras preferencias sean sensibles a la riqueza de que podemos llegar a disponer, y en este sen- 
tido es que, al encontrarse las preferencias entrelazadas con juicios sobre nuestra disponibilidad de bienes, es posible hablar de una adaptabilidad de las preferencias a los recursos que pueden llegar a controlarse. Esto significa que alguien puede llegar a tomar la decisión de empobrecerse por buscar realizar un cierto tipo de ambición, siendo un ejemplo paradigmático de este caso lo que los grandes artistas han hecho en su juventud. Pero aun en estos casos es necesario tener una cierta expectativa de la pobreza que puede llegar a sufrirse, porque no es posible llegar a establecer preferencias estables sin tener algún referente de este tipo ${ }^{13}$.

De esta manera, Dworkin presenta sujetos que son racionales en tanto que estructuran un plan de vida coherente, y que, en consecuencia, pueden revisar y adaptar ese plan de vida. Esto supone reconocer que un plan de vida o una concepción del bien se encuentran condicionados por una serie de factores fluctuantes; por lo tanto, deben ser asumidos como una empresa falible sujeta a considerables modificaciones e incluso a su abandono. Esta idea de revisabilidad inherente, tanto a un plan de vida o a una concepción del bien como a una teoría, es expresado por Buchanan en el siguiente principio. «R: Uno debería, ceteris paribus, mantener una actitud de revisabilidad crítica hacia la propia concepción del bien (o plan de vida) y una actitud de apertura hacia concepciones competentes» ${ }^{14}$.

Este principio de revisabilidad se compromete con la postura de que la evaluación de las concepciones del bien o los planes de vida es una actividad racional. Incluso en su versión más débil, tal principio implica coherencia en el sistema de creencias mantenidas, y esto exige que revisemos algunos de nuestros juicios de valor. De acuerdo con esto, será necesaria una actitud de revisabilidad y, en consecuencia, de adaptabilidad, hacia nuestros propios juicios de valor y de ahí hacia nuestra concepción del bien.

Esta concepción de racionalidad, que es inherente a la propuesta de Dworkin, es a grandes rasgos compartible, pero no supera una limitación considerable que expondré a continuación. De acuerdo con lo presentado, en la perspectiva de Dworkin no habría posibilidad de que alguien tuviera ambiciones, preferencias, o creencias que no fuesen racionales, ya que éstas se encuentran fuertemente entrelazadas en un proceso racional que posibilita la adaptabilidad y la revisabilidad a los recursos que se controla o a los que se tiene expectativa de controlar. Todo este proceso se lleva adelante bajo el supuesto de la coherencia en la totalidad de creencias, preferencias, valores, etc., que tienen los individuos. Nuestro cuestionamiento se centra en que muchas veces los sujetos tienen comportamientos que Dworkin calificaría de racionales porque son coherentes con su universo de creencias, pero que difícilmente puedan ser aceptables por una perspectiva de justicia. En particular, me refiero a la conformación de preferencias que serían consecuencia de aquellos procesos que son caracterizados como formación de preferencias adaptativas, por los cuales se da el ajuste de las voliciones a las posibilidades. Éste es un proceso causal que ocurre de manera no conciente y que hace que detrás de la adaptación se presente la tendencia a eludir la frustración que se siente al experimentar deseos que no pueden satisfacerse ${ }^{15}$. En palabras de Nussbaum, «las circunstancias confinan la imaginación» ${ }^{16}$. Por ejemplo, podemos considerar el caso de un marginal que ha sufrido un proceso de adaptación de creencias a su situación de carencia extrema y en virtud de su condición marginal estructura su plan de vida. Esas decisiones pueden involucrar que sus hijos no ingresen al sistema educativo, porque en su casa pueden colaborar en el trabajo doméstico, por ejemplo, cuidar a los hermanos, rea- 
lizar pequeñas tareas, etc.; por el contrario, si fueran a la escuela perderían una gran cantidad de horas para en última instancia continuar reproduciendo esa forma de vida. También nuestro marginal puede decidir no buscar trabajo más allá de las tareas que realiza, por ejemplo, reciclando basura, ya que todo intento de ese tipo lo ha llevado a él y a quienes comparten su universo a continuar en la misma situación. Por lo tanto, de acuerdo a una serie de circunstancias que han afectado la vida de nuestro protagonista, tendríamos que el decidir no buscar empleo formal o decidir que sus hijos no accedan a ningún tipo de instrucción formal, son decisiones racionales, es decir, son decisiones informadas, coherentes con su sistema de creencias y que incluso pueden ser producto de un proceso de revisabilidad y readaptación. Este proceso puede ser entendido como motivado en última instancia por esta búsqueda de la reducción de la frustración o simplemente como un proceso de readaptación de creencias que tiende a optimizar el proyecto de vida de nuestro afectado. En ambos casos indistintamente la situación es intolerable desde una perspectiva de justicia, pasando a ser cuestionable la responsabilidad que tendría este sujeto.

Ante este tipo de casos Dworkin diría que el sujeto, en tanto que racional, es responsable de sus acciones, y en virtud de ello no tendría derecho a ningún tipo de compensaciones. Esto está pautando la insuficiencia del criterio para atribuir responsabilidad, porque si algo queda claro, casi de forma intuitiva, es que este tipo de casos demanda un tratamiento que asegure cierto tipo de compensaciones.

\section{III}

Para poder explicar estos problemas partiré de lo que Adela Cortina denomina el factum de la autonomía, según el cual «contar con sujetos hasta cierto punto autónomos, es condición de sentido y validez de buena parte de nuestra vida (...)» ${ }^{17}$. Este factum puede ser entendido en base a la idea de personalidad moral ${ }^{18}$, por lo que los sujetos contarían, como elemento determinante de su condición, con las capacidades de tener un sentido de justicia y albergar una concepción del bien. El sentido de justicia es la capacidad para entender, aplicar y actuar de acuerdo a una concepción pública de justicia que opera definiendo los términos equitativos de la cooperación social. Por su parte, la capacidad para concebir el bien consiste en poder formar, revisar y perseguir racionalmente una concepción del propio bien.

Partiendo de la autonomía como trasfondo, introduciré la distinción entre autonomía potencial y autonomía plena, donde esta última no estaría caracterizada por el ejercicio máximo de la autonomía, sino por aquellos mínimos que nos permitan reconocer en los sujetos estas dos capacidades de la personalidad moral. Por su parte, la autonomía potencial sería la característica de aquellos sujetos que no han llegado a un nivel mínimo del desarrollo de estas dos capacidades.

El problema del derecho a las compensaciones por parte de los sujetos tiene que ver con circunstancias que están fuera de su control y que limitan el ejercicio de su autonomía. La autonomía sería potencial en todos aquellos casos en que las capacidades requeridas para el ejercicio de la autonomía se encontrasen limitadas. Cabe aclarar que me estoy refiriendo no solamente a las capacidades de la personalidad moral, sino a todas aquellas que pueden limitar el pleno ejercicio de estas últimas. Una discapacidad, por ejemplo, no es identificable como la carencia de una de las capacidades de la personalidad moral, pero, sin embargo, es un impedimento significativo para una de ellas, porque una discapacidad vuelve más dificultoso el llevar adelante un plan de vida. Por su parte, la autonomía plena se presentaría cuando 
el individuo está en pleno ejercicio de sus capacidades, tanto de las de la personalidad moral, como de aquellas que contribuyen al ejercicio de estas últimas. El intento de contrarrestar estas circunstancias por parte de los arreglos de justicia tiene como meta el poder llevar a los sujetos desde esa autonomía potencial a la plena.

A partir de esta distinción es posible enfrentar con herramientas diferentes el problema de la responsabilidad por las propias decisiones, y en virtud de ello discriminar qué resultados merecen compensación y cuáles no.

En el caso analizado, el único criterio para discriminar la responsabilidad de un sujeto era el de la racionalidad. Pero esto pasa por alto el hecho de que un marginal que no sea plenamente autónomo puede realizar elecciones que, aunque racionales, no representen sus mejores intereses. Esto se debe a que, en virtud de su autonomía potencial, no ha desarrollado las capacidades necesarias para ejercer su autonomía. En esta situación, como ya he indicado, habría coherencia entre creencias y preferencias, pero eso no libera el problema, sino que lo vuelve más complejo.

La complejidad se asienta en que el criterio de la racionalidad manejado por Dworkin no es suficiente para discriminar entre aquellas preferencias y elecciones de las que un sujeto sería responsable y aquellas que no. La racionalidad no es un criterio suficiente desde el punto de vista de la justicia para discriminar entre aquello de lo que alguien es responsable y aquello de lo que no. Es decir, si la motivación para el tratamiento de la responsabilidad se encuentra en la búsqueda de criterios que permitan compensar a aquellos que han sufrido situaciones deficitarias en términos de recursos sin ser responsables por ello, entonces se hace necesario construir un criterio más preciso.

La dificultad se encuentra en determinar hasta qué punto una sociedad que par- te del factum de la autonomía, admite que esta última se encuentre disminuida. Buscando dar respuesta a esto último es que he presentado la distinción entre autonomía potencial y autonomía plena. Al realizar esta distinción, la autonomía es presentada como un concepto continuo que tendría un umbral que marcaría el pasaje de un estadio a otro. Algunos autores, como Beauchamp y Childress ${ }^{19}$, en el ámbito de la bioética, realizan una distinción similar colocando el umbral en lo que ellos denominan competencia. Lo importante es aproximarnos a delimitar aquello que hace que alguien pase el umbral que separa a la autonomía potencial de la autonomía plena, y en tal sentido creo que se puede afirmar que existe un conjunto de capacidades que determinan el ejercicio de las capacidades de la personalidad moral en términos de mínimos, y tal desarrollo es el que permitiría pasar de un estadio a otro.

Puede sostenerse que para que un sujeto pueda llegar al ejercicio mínimo de las dos capacidades de la personalidad moral es necesario, por ejemplo, que pueda deliberar y decidir bajo un transfondo de un conjunto de valores y fines. Esto a su vez puede expandirse en que el individuo pueda recibir y reconocer la información relevante, que también pueda razonar sobre alternativas y ordenarlas de acuerdo a criterios de prioridad, y que pueda elegir y dar cuenta del proceso por medio de razones.

Estos supuestos, que hacen a un sujeto en estadio de autonomía plena, demandarán a su vez las condiciones para que se pueda alcanzar tal estadio. En función de eso es que la condición de autonomía puede operar como criterio para el diseño de políticas que apunten al desarrollo de estas capacidades.

Pero existe un problema, que se presenta también en el área de la bioética ${ }^{20}$, y es aquel que tiene que ver con la movilidad de la competencia; es decir, el criterio 
que divide a un sujeto con autonomía potencial de uno con autonomía plena puede ser más o menos exigente, en función del caso de que se trate. Por ejemplo, probablemente debido a que la preservación de la vida se presenta como un objetivo menos problemático que la adquisición de conocimiento en un sistema educativo formal, las decisiones acerca de la cobertura sanitaria parecen ser menos exigentes que las que tienen que ver con el proceso educativo. La asistencia a un proceso educativo formal exige ciertos hábitos y disciplina, que de no estar instaurados en la familia del afectado culminarán con el abandono del sistema. A su vez, se presenta una demanda aún mayor, y es que los procesos educativos exigen un costo personal que tiene una recompensa a largo plazo, y la percepción de este tipo de recompensas y la adopción del costo que exige el proceso educativo es una condición más de exigencia. Por su parte, la cobertura sanitaria solamente requiere el deseo de preservar la propia vida y la de los afectados que se expresa a través de una decisión, que, existiendo un sistema de salud pública, no aparejaría mayores dificultades.

Estos dos tipos de casos demandarían diferentes exigencias a la autonomía, a lo que se podría responder que el poder público podría tomar el más alto de estos criterios para diseñar las políticas públicas. Si bien este criterio sería el más exigente de las posibles situaciones de decisión, no apuntaría al ejercicio más alto de la autonomía plena, sino que solamente aseguraría en términos de mínimos las dos capacidades de la personalidad moral, y estas capacidades habilitarían a resolver los diferentes problemas de decisión que se le pueden plantear a un individuo. No sería posible hablar de autonomía plena si algún tipo de decisión no fuese alcanzable, aunque claro está que esto de ninguna manera significa el ejercicio máximo de la autonomía.
De esta forma, se apuntaría a asegurar políticas compensatorias y de desarrollo de capacidades orientadas a que universalmente se alcance el estadio de autonomía plena que pueda responder a las circunstancias con mayor exigencia.

\section{IV}

Una lista de capacidades que permitan situar el umbral que divide la autonomía potencial de la plena se vuelve necesario, y para ello apelaré a la tarea ya realizada por Nussbaum, quien parte para ello de un conjunto de capacidades que son características determinantes de la humanidad, es decir, que una vida en ausencia de ellas estaría tan empobrecida que no sería una vida humana en ningún sentido. Esto establece un primer nivel de evaluación, que no supone que la presencia de estas capacidades determine una vida buena. Por eso es necesario, sostiene Nussbaum ${ }^{21}$, distinguir dos umbrales, el primero de los cuales sería aquel que hace a la «capacidad para funcionar», por debajo del cual una vida estaría tan disminuida que no sería una vida humana. Un segundo umbral sería superior al primero, lo que asegura el reconocimiento de la humanidad, pero si la capacidad para funcionar no superase este segundo umbral, no podría sostenerse que esa vida es una buena vida.

Es imprescindible aclarar que Nussbaum, con «buena vida», no está refiriéndose a ninguna concepción del bien en particular o concepción comprehensiva, sino a aquellas capacidades que permitirán a un individuo llevar adelante una vida digna. Volviendo a nuestra terminología, puede sostenerse que el segundo umbral estaría delimitando la autonomía potencial de la plena. Como muy claramente hace notar Nussbaum, el moverse desde la «vida humana» a «la buena vida humana» puede generarse por los propios afectados, es decir, que una vez que se ha superado el 
primer umbral es posible que los afectados, en virtud de sus propias capacidades, puedan por ellos mismos superar el segundo umbral. Sin embargo, esto es así en lo que tiene que ver con algunas capacidades, pero no todas. Un caso paradigmático de capacidades que posibilitan el pasaje de un umbral a otro por el propio afectado es tal vez el del razonamiento práctico, mientras que en lo que hace a salud y nutrición la distancia entre los dos umbrales es mayor, ya que esta diferencia tiene que ver con recursos sobre los que los afectados no tienen control.

Especificando lo requerido por el segundo umbral, es decir, el del desarrollo del conjunto de capacidades que no solamente nos permiten identificar a alguien como ser humano, sino que posibilitan que ese individuo pueda llevar adelante una «buena vida», es mi posición que las capacidades para funcionar serían las que asegurarían el pasaje de lo que he denominado autonomía potencial a autonomía plena. Nussbaum presenta la siguiente lista:

1. Ser capaz de vivir una vida completa, no morir prematuramente.

2. Ser capaz de tener buena salud, de estar bien alimentado, de tener adecuada vivienda, de tener oportunidades de satisfacción sexual, de moverse hacia diferentes lugares.

3. Ser capaz de evitar el dolor innecesario y de tener experiencias placenteras.

4. Ser capaz de usar los cinco sentidos; ser capaz de imaginar, de pensar, y de usar la razón.

5. Ser capaz de experimentar apego a cosas y personas fuera de nosotros mismos; en general, amar, llorar la muerte de alguien, extrañar y sentir gratitud.

6. Ser capaz de formar una concepción del bien y reflexionar críticamente acerca de la planificación de nuestra vida.

7. Ser capaz de vivir por y con otros, de reconocer y mostrar consideración por otros seres humanos, de tomar parte en interacciones sociales y familiares.

8. Ser capaz de sentir consideración por y en relación con animales, plantas y la naturaleza.

9. Ser capaz de humor, de juego, y de disfrutar actividades recreativas.

10. Ser capaz de vivir la propia vida, y no la de alguien más, en el contexto que le ha tocado ${ }^{22}$.

En este conjunto de capacidades básicas, que una vez que fuesen adquiridas en forma mínima superarían el umbral que colocaría al individuo en un estado de autonomía plena, dos de ellas ocupan un lugar central y estructurante del resto del conjunto $^{23}$. Tales capacidades son la del razonamiento práctico (4 en la lista), y la de la afiliación (7 en la lista), debido a que son las únicas capacidades que planifican y ordenan la forma en que las otras capacidades entran en juego, y no solamente eso, sino que su intervención hace que el resto de las capacidades sean específicamente humanas. De acuerdo con esto, la alimentación, la satisfacción del deseo sexual, etc., son diferentes a idénticas capacidades de otros animales, básicamente porque la razón práctica permite que los seres humanos puedan elegir la regulación de las mismas, y también porque esas capacidades no son ejercidas en solitario, sino como seres humanos que se relacionan con otros a través de lazos de mutua atención y consideración.

Las características de estas dos capacidades estructurantes del conjunto son tales que determinan directamente las dos capacidades de la personalidad moral: el razonamiento práctico lo hace con respecto a la capacidad de albergar y perseguir una concepción del bien, y la afiliación lo hace con respecto a la capacidad para albergar un sentido de justicia. Desde mi punto de vista he establecido la distinción entre autonomía potencial y plena, no solamente porque considero que el razona- 
miento práctico y la afiliación juegan un rol estructurante en el conjunto de capacidades básicas, sino porque, a diferencia de Nussbaum, creo que es lo determinante de la condición de ser humano. Las consecuencias de reducir el peso de estas capacidades distintivas impactarían directamente en las políticas públicas, afectando las posibilidades de acceder a procesos de construcción de autonomía por parte de los afectados. Debido a esto es que la distinción entre autonomía potencial y plena se presenta como más apropiada para describir dos estados diferentes de un continuo que caracteriza a lo propio, lo determinante del sujeto.

La idea de potencialidad, determinada por un bajo nivel en el desarrollo de capacidades que permitan llevar adelante las funciones propias de un sujeto autónomo, significa que estos individuos, bajo condiciones de educación y compensación de cierto tipo, necesariamente alcanzarían el estado de autonomía plena. Esta relación entre potencialidad y plenitud es la que genera el deber de que las políticas públicas apunten a superar esa brecha, si es que el supuesto que asegura el tratamiento igualitario sigue siendo vinculante.

Es necesario reiterar que la lista es una lista abierta y general, que permite variaciones en su aplicabilidad, tanto por las múltiples especificaciones de cada uno de los componentes de la lista, como de las distintas sociedades a las que se aplicaría. Esta variabilidad no afecta al conjunto de capacidades básicas, sino que lo especifica, de tal manera que, por ejemplo, distintas sociedades pueden requerir montos sustancialmente diferentes para poder alcanzar iguales capacidades. En virtud de esto, el umbral que distingue autonomía potencial de autonomía plena varía con la misma lógica, pero, como ya he indicado, el umbral que tiene que ver con las capacidades del razonamiento práctico, en la medida que cumple un papel vital en la constitución del sujeto autónomo, debe colocarse en aquel nivel que posibilite dar respuesta a todos los requerimientos de la autonomía.

\section{V}

Este conjunto de capacidades no difiere mucho de aquello que podría ser incluido en una extensión del mecanismo del mercado hipotético de seguros en Dworkin, ya que si este mecanismo tiene como objetivo compensar a nivel de mínimos en todos aquellos aspectos que obstaculicen la persecución de un plan de vida, entonces se presenta como parte de la lógica del mecanismo la posibilidad de extenderlo a otras áreas.

En ese sentido, si se establece la línea divisoria para aquellas decisiones de las que son responsables los individuos en aquellas a las que se llega por un ejercicio mínimo de la autonomía plena, entonces casos como los del ejemplo del marginal que decide no enviar a su hijo al colegio deberían dar lugar a compensaciones en recursos, porque este tipo de sujetos no son sujetos plenamente autónomos y, por lo tanto, requieren ser llevados a ese estadio para poder ser absolutamente responsables de sus decisiones. De acuerdo con esto, debería asegurarse que los sujetos accedieran a todos aquellos procesos sociales que les permitiesen adquirir la autonomía plena en términos de mínimos. Una vez adquirida, los mecanismos y criterios utilizados por Dworkin para discriminar a quién compensar y a quién no se presentan sumamente efectivos.

De inmediato surgen algunos problemas acerca de cómo identificar a sujetos que son potencialmente autónomos. Ya hemos visto que la racionalidad no está disponible como criterio, ya que la formación de preferencias adaptativas cumpliría con la condición de ser racional en términos de coherencia. De ahí que deba apelarse a las condiciones en que han sido 
tomadas tales decisiones; seguramente estas condiciones limitan considerablemente la autenticidad de la decisión, ya que en casos como el de nuestro ejemplo, puede ser que al tomar sus decisiones los afectados no hayan tenido acceso a toda la información o a buena parte de la información relevante para tomarlas, así como tampoco se tiene la certeza de que su capacidad de evaluación y deliberación se encuentren en el nivel requerido por el ejercicio pleno de la autonomía. En este punto considero que el criterio que maneja Cohen de la disponibilidad de información para establecer la autenticidad de una elección es bastante restrictivo, y cabe señalar que recientemente Dworkin ha prestado mayor atención a este punto y ha colocado el manejo de información como una condición relevante para la realización de juicios con vistas a un mercado hipotético de seguros ${ }^{24}$.

Tanto la posición de Cohen como la del reciente Dworkin se presentan insuficientes, porque no consideran la posibilidad de que, frente a suficiente información, el individuo no esté en condiciones de procesarla; por lo tanto, la identificación de aquellos sujetos que sean potencialmente autónomos deberá apelar tanto a la cantidad de información que se encuentra a su disposición como a las capacidades para procesarla, por ejemplo, deliberación, evaluación de mejores posibilidades, etc.

Con esto quiero decir que si un profesional universitario decide renunciar a su trabajo y dedicar su vida a vivir en la pobreza reciclando basura, probablemente concluyamos que este individuo encuentra algún tipo de valor en esta forma de vida, es decir, es la decisión tomada por alguien que se encuentra en el ejercicio de su autonomía plena. Pero si el caso es el de un marginal que decide no buscar trabajo formal y dedicar su vida a reciclar basura, entonces probablemente estemos en presencia de un caso en el que su decisión no sea el producto del ejercicio de su auto- nomía plena. Teniendo como antecedente un idéntico acceso a la información y el mismo desenlace, los procesos de decisión de estas dos personas han sido radicalmente diferentes, y tal diferencia se encuentra en que las capacidades desarrolladas por el profesional universitario lo colocan en una situación en la que elige entre diferentes posibles cursos de acción uno en particular, mientras que en el caso del marginal las posibilidades de elección son nulas, puesto que, si bien formalmente puede tener diferentes posibles cursos de acción, sus capacidades no le permiten procesar una elección de ese tipo.

En definitiva, una sociedad que suponga el factum de la autonomía no puede dejar de hacerse cargo de la construcción de la autonomía de los sujetos, porque si lo hace estaría renunciando a tratar a todos sus ciudadanos como iguales; por lo tanto, una sociedad con tal supuesto debe asegurar la igual consideración de sus ciudadanos al nivel más básico, es decir, en la posibilidad de actuar como sujetos autónomos.

Volviendo al criterio manejado por Dworkin para atribuir responsabilidad, y en particular a su concepción de justicia, es posible defender una interpretación de su propuesta que cuente como presupuesto con la distinción entre autonomía potencial y plena. A partir de tal distinción es posible interpretar el mecanismo del mercado hipotético de seguros como una forma de llevar a los sujetos del estadio de autonomía potencial al de autonomía plena. Por lo tanto, dentro de lo que sería compensable por el mercado hipotético de seguros se encontraría todo lo que es parte de las circunstancias del sujeto y que le impide ser un sujeto plenamente autónomo.

Por otra parte, a nivel de la dimensión de las circunstancias, en casos problemáticos como aquellos que se sitúan en el límite de gustos caros y características personales, se puede recurrir al propio sujeto, y de acuerdo a cómo percibiera esa carac- 
terística es que se demarcaría entre discapacidad o preferencia. Si el sujeto prefiriera no tener esa característica, si, por ejemplo, se habría asegurado contra la posibilidad de adquirirla si el seguro hubiera estado disponible, entonces tal característica personal sería parte de sus circunstancias y, en consecuencia, considerada como una discapacidad. Pero si la considerara como una característica que preferiría seguir teniendo, entonces entraría dentro del campo de la personalidad y sería calificada como preferencia.

Tomando la distinción entre autonomía potencial y plena, en el caso de las elecciones y la atribución de responsabilidad no es posible apelar al sujeto mismo como criterio discriminatorio, como se hace en el caso de preferencias y discapacidades. La razón es bastante obvia, y es que un sujeto potencialmente autónomo no puede enajenarse y evaluar si sus elecciones son autónomas o no; en todo caso, si esto es posible, es a partir del momento en que es un sujeto plenamente autónomo y puede tomar distancia de su posición y evaluar. Pero en este caso, su juicio sería irrelevante porque el problema que necesita resolverse es exclusivamente a nivel de la autonomía potencial.

El criterio para discriminar entre autonomía potencial y plena y, en consecuencia, entre elecciones responsables y aquellas que no lo son, se asienta en un conjunto de capacidades mínimas a desarrollar que permiten discriminar entre un tipo de sujeto y otro, y que posibilitarían compensar a los sujetos potencialmente autónomos a través del mecanismo del mercado hipotético de seguros, extendiéndolo a todas aquellas capacidades necesarias para llevar al sujeto del estado de autonomía potencial al de plena. La lista elaborada por Nussbaum se presenta como una guía sumamente útil para tal tarea.
Cf. R. Dworkin, «Why Liberals Should Believe in Equality», The New York Review of Books, 3 de febrero de 1983, p. 32. Este esbozo de distribución de recursos es ampliamente desarrollado en R. Dworkin, «What Is Equality? Part II: Equality of Resources», Philosophy and Public Affairs, núm. 10, 1981, pp. 283-345.

2 Cf. R. Arneson, «Equality and Equal Opportunity for Welfare», Philosophical Studies, vol. 56, núm. 1, mayo 1989, pp. 77-93; G. Cohen, «On the Currency of Egalitarian Justice», Ethics, 99, núm. 4, 1989, pp. 906-944; J. Roemer, «A Pragmatic Theory of Responsability for the Egalitarian Planner», Philosophy and Public Affairs, vol. 22, núm. 2, primavera de 1993. Reimpreso en J. Roemer, Egalitarian perspectives. Essays in philosophical economics, Cambridge, Cambridge University Press, 1994, pp. 179-196; K. Lippert-Rasmussen, «Egalitarianism, Option Luck, and Responsibility», Ethics, núm. 111, abril 2001, pp. 548-579; E. Anderson, «What Is the Point of Equality», Ethics, núm. 109, enero 1999, pp. 287-337.

${ }^{3}$ Aristóteles, Ética Nicomáquea, Madrid, Gredos, 1993, 1110b 30-1111a 3.
${ }^{4}$ Cf. R. Arneson, op. cit.

5 Cf. ibid., p. 80.

${ }^{6}$ Cf. G. Cohen, op. cit., p. 919.

7 Cf. R. Dworkin, «Equality and Capability», en Sovereign Virtue, Londres, Harvard University Press, 2000, p. 287.

${ }^{8}$ Cf. R. Dworkin, «Equality of Resources», p. 302; «Equality and Capability», p. 291.

${ }^{9}$ Cf. G. Cohen, op. cit., p. 929.

${ }^{10}$ Cf. ibid., p. 935.

${ }^{11}$ Cf. R. Arneson, op. cit., p. 92.

${ }^{12}$ Cf. R. Dworkin, «Equality and Capability», p. 293.

${ }_{13}$ Cf. ibid., p. 293.

${ }_{14}$ A. Buchanan, «Revisability and Rational Choice», Canadian Journal of Philosophy, vol. V, núm. 3, noviembre 1975, p. 399.

15 Cf., J. Elster, Uvas amargas. Sobre la subversión de la racionalidad, Barcelona, Península, 1988, p. 42.

${ }^{16}$ M. Nussbaum, «Human functioning and Social Justice. In Defense of Aristotelian Essentialism», Political Theory, vol. 20, núm. 2, mayo 1992, p. 230. 
17 A. Cortina, Ética aplicada y democracia radical, Madrid, Tecnos, 1993, p. 127.

${ }^{18}$ Cf. J. Rawls, «Kantian Constructivism in Moral Theory», The Journal of Philosophy, LXXVII, 1980, pp. 512-572. Traducción castellana, «El constructivismo kantiano en la teoría moral», en Justicia como equidad. Materiales para una teoría de la justicia, Madrid, Tecnos, 1986, pp. 145-146.

${ }^{19}$ Cf. T. Beauchamp y J. Childress, Principles of Biomedical Ethics. Fourth Edition, Nueva York/Oxford, Oxford University Press, 1994, p. 135.
${ }^{20}$ Cf. J. Drane, «Competency to Give an Informed Consent. A model for Making Clinical Assessments», JAMA (Journal of the American Medical Association), vol. 252, núm. 7, 1984, pp. 925-927, y «The Many Faces of Competency», The Hastings Center Report, vol. 15, núm. 2, marzo-abril 1985, pp. 17-21.

${ }^{21}$ Cf. M. Nussbaum, op. cit., p. 221.

22 Cf. ibid., p. 222.

${ }^{23}$ Cf. ibid., p. 222.

${ }^{24}$ Cf. R. Dworkin, «Justice and the High Cost of Health», en Sovereign Virtue, p. 312. 